\title{
Atenção domiciliar: perfil assistencial de serviço vinculado a um hospital de ensino
}

\section{I ${ }^{1}$ Ana Carolina de Oliveira Jeronymo Neves, ${ }^{2}$ Clarissa Terenzi Seixas, ${ }^{3}$ Angélica Mônica Andrade, ${ }^{4}$ Edna Aparecida Barbosa de Castro I}

Resumo: Objetivos: Descrever o perfil dos usuários e cuidadores atendidos pelo Serviço de Atenção Domiciliar; verificar associaçôes e correlaçôes entre as variáveis. Método: Estudo do tipo documental, quantitativo, realizado entre maio e novembro de 2017. A análise dos dados foi feita por meio de estatística básica, teste qui-quadrado e coeficiente de correlação de Pearson. Resultados: Dos 46 usuários, a maioria era idosa, do sexo feminino $(71,74 \%)$, com renda de até dois salários mínimos (65,22\%), dependentes para o autocuidado (89,13\%). A variável "grau de dependência" teve associação estatisticamente significante com o sexo ( $p=0,025)$, o motivo de inclusão $(p=0,003)$, especialidade médica $(\mathrm{p}=0,013)$, classificação no $\mathrm{SAD}(\mathrm{p}<0,001)$ e a presença de cuidador familiar $(\mathrm{p}=0,017)$. Demandavam 7,46 equipamentos de saúde, sendo que $70 \%$ eram arcados pela família; e 6,78 procedimentos de enfermagem, dos quais $52 \%$ eram realizados pelo cuidador. Houve diferença estatisticamente significante entre os custos e gastos assumidos pelas famílias ( $\mathrm{p}<0,001)$, o número de equipamentos de saúde ( $<<0,001)$ e o número de procedimentos de enfermagem demandados $(\mathrm{p}<0,001)$. Sobre os cuidadores, a maioria era do sexo feminino (85,71\%), com idade média de 62,3 anos, $50 \%$ eram filhos(as) e 23,81\% esposos(as). Conclusão: Funçōes, responsabilidades, custos e gastos são transferidos às famílias, onerando o cuidado domiciliar.

> Palavras-chave: assistência domiciliar; cuidadores; necessidades e demandas de serviços de saúde; custos de cuidados de saúde.

\author{
1 Universidade Federal de Juiz \\ de Fora. Juiz de Fora-MG, Brasil \\ (anacarol.jeronymo@gmail.com). \\ ORCID: 0000-0002-3616-2904 \\ ${ }^{2}$ Universidade do Estado do Rio \\ de Janeiro. Rio de Janeiro-RJ, \\ Brasil (claseixas@gmail.com). \\ ORCID:0000-0002-8182-7776 \\ ${ }^{3}$ Universidade Federal do Rio \\ de Janeiro. Rio de Janeiro-RJ, \\ Brasil (angelicamonica.andrade@ \\ gmail.com). \\ ORCID: 0000-0003-2684-1134 \\ ${ }^{4}$ Universidade Federal de Juiz \\ de Fora. Juiz de Fora-MG, Brasil \\ (ednabdecastro@hotmail.com). \\ ORCID: 0000-0001-9555-1996
}

Recebido em: 25/05/2018 Revisado em: 20/02/2019 Aprovado em: 27/02/2019 


\section{Introdução}

O envelhecimento e a cronificação de doenças nas populaçôes têm levado os governos a repensarem o modelo de atençáo em saúde, provendo políticas públicas específicas, o financiamento de estruturas de apoio, a avaliação e o monitoramento de serviços, com destaque para o resgate do domicílio como ambiente terapêutico e a reorganização da Atenção Domiciliar (AD) como estratégia de atenção no âmbito do Sistema Único de Saúde (SUS) (WACHS et al., 2016; BRAGA et al., 2017).

Em 2011, após revisóes do conjunto de legislaçóes e normativas que orientavam a AD no Brasil, foi instituída a Política Nacional de Atenção Domiciliar (PNAD), pela Portaria no 2029, que criou o Programa Melhor em Casa (PMC) como subsídio à implantação dos Serviços de Atenção Domiciliar (SAD) pelos municípios (BRASIL, 2011). Desde então, a $\mathrm{AD}$ tem se configurado como um espaço não tradicional de atenção à saúde, em que os profissionais, juntamente com o(s) cuidador(es), cuidam de usuários elegíveis ao atendimento em casa. O domicílio ganha dimensão de ambiente peculiar, singular, possibilitando que a assistência esteja centrada no usuário e em sua família, por meio da estruturação, gestão e execução de um projeto terapêutico singular (PTS), juntamente com a Atenção Primária à Saúde (APS) (ANDRADE et al., 2017).

$\mathrm{Na}$ AD, a assistência ofertada organiza-se em três modalidades: AD1, AD2 e AD3. A definição da modalidade assistencial relaciona-se à densidade tecnológica, às necessidades de saúde, à periodicidade das visitas, à intensidade do cuidado multiprofissional e ao uso de equipamentos. Os critérios de elegibilidade e inclusão a uma delas podem ser clínicos e administrativos, normatizados pela Portaria no 825/2016. Consideram-se elegíveis à AD1 aqueles cujo estado de saúde esteja estável, possibilitando que a assistência seja realizada no domicílio, por cuidadores, sob a responsabilidade de acompanhamento mensal pela APS. À AD2 elegem-se aqueles com afecçóes agudas ou crônicas agudizadas, com necessidade de cuidados intensificados e sequenciais, minimamente semanais, por equipe multiprofissional, e, caso demandem equipamentos e procedimentos de maior complexidade, estes passarão à modalidade de AD3. (BRASIL, 2016).

Em um SAD, enfermeiro e técnicos de enfermagem integram-se a outros profissionais na Equipe Multiprofissional de Atenção Domiciliar (EMAD). A atuação do enfermeiro na $\mathrm{AD}$ requer habilidades e conhecimentos próprios, pois 
este, frequentemente, é responsável por coordenar o plano de cuidados no domicílio de forma compartilhada com a família, centrando o cuidado nas necessidades e demandas do usuário. O processo de trabalho do enfermeiro, nesse cenário, também inclui processos de educação em saúde e suporte aos cuidadores familiares, assim como orientação e supervisão do trabalho dos técnicos de enfermagem e execução de procedimentos de maior complexidade (ANDRADE et al., 2017).

Os $\mathrm{SAD}$ atendem usuários, em sua maioria, idosos em processo de adoecimento secundário a doenças crônicas, acamados, parcialmente ou totalmente dependentes para o autocuidado (CARNAÚBA et al., 2017; NEVES; CASTRO; COSTA, 2016). Os cuidadores, em sua maioria, são familiares do sexo feminino, idosas, filhas ou esposas, residentes no mesmo domicílio, com baixa escolaridade, conhecimento e treinamento insuficientes para o desempenho de sua função (LOUREIRO; FERNANDES, 2015). No ambiente domiciliar, os cuidadores com ou sem vínculo familiar com o usuário o auxilia em suas necessidades e atividades da vida cotidiana, além de se envolver com ele, oferecendo atenção e zelo (BRASIL, 2016; MOCELIN et al., 2017).

Um estudo brasileiro identificou que, assim como em diferentes sociedades e culturas, o cuidador domiciliar informal guarda relação com o tipo de arranjo familiar, mostrando-se prevalente nos mais numerosos, onde é assumido por "parente remunerado ou empregada doméstica”, ou seja, por indivíduos sem formação profissional em uma instituição de ensino, em detrimento do cuidador contratado. Todavia, os autores alertam para a mudança nos arranjos familiares, marcada pela redução do número de membros, sobretudo pelo "aumento do número de casais sem filhos” (LIMA-COSTA et al., 2017; SOLÉ-AURÓ; CRIMMINS, 2014).

Depreende-se a possibilidade deste aspecto vir a constituir-se em um fator de risco para a manutenção, ou mesmo para a precarizaçáo do cuidado domiciliar a esse grupo populacional, já considerado frágil, reforçando a premente necessidade de se investir em pesquisas e em políticas públicas de apoio ao cuidador familiar e de enfrentamento à crescente realidade de entrada do cuidador informal remunerado no contexto do trabalho em saúde, exercendo essa função sem a adequada formação por instituições de ensino (VAZ; SANTOS; FERRAZ, 2018).

O processo de transição do cuidado dispensado no hospital para o domicílio altera a dinâmica e as relaçóes familiares, sendo que a gestão do cuidado é parcial 
ou integralmente transferida para a família, ocasionando a sua sobrecarga física e emocional (CASTRO et al., 2018). Quando o cuidado é assumido apenas por um familiar, a atividade torna-se ainda mais desgastante, pois, muitas vezes, este é privado de satisfazer as suas próprias necessidades, comprometendo sua saúde, causando isolamento e perdas sociais (MOCELIN et al., 2017).

Somados às limitaçôes de conhecimento, ao desgaste emocional e ao desempenho de novos papéis, estudos apontam como dificuldades vivenciadas pelo cuidador o enfrentamento da burocracia no SUS e outros entraves, como a falta de apoio profissional no âmbito domiciliar, a indisponibilidade de materiais e de equipamentos, entre outros (ANJOS; ZAGO, 2014; NEVES; CASTRO; COSTA, 2016).

Segundo Braga e colaboradores (2017), há um descompasso na AD entre a lógica sob a qual se organiza a oferta, visando à racionalização de custos e não à melhor alocação dos recursos disponíveis, e o atendimento das reais demandas e necessidades de saúde da população. Esses custos podem ser classificados em diretos, indiretos e intangíveis. $\mathrm{O}$ custo direto configura-se naquele que é possível atribuir diretamente ao procedimento ou atividade (MESQUITA et al., 2005); o indireto é aquele que náo se pode atribuir diretamente a cada tipo de bem, no momento de sua ocorrência, sendo apropriado aos portadores finais mediante o emprego de critérios predeterminados e vinculados a causas correlatas (OLIVEIRA; SANTOS; SILVA, 2014). Já os intangíveis são de difícil avaliação, pois englobam aspectos físicos, emocionais e sociais e representam as mudanças na qualidade de vida e as consequências da patologia e do seu tratamento (OLIVEIRA; SANTOS; SILVA, 2014; BRASIL, 2008).

Os custos para a família têm impacto diferenciado, considerando os diferentes níveis de poder aquisitivo, sendo que este impacto se torna ainda maior nas famílias de baixa renda, o que pode dificultar a continuidade do cuidado no contexto domiciliar (FALLER et al., 2012). Além disso, destaca-se que o custo de cuidar em casa ultrapassa o âmbito financeiro, uma vez que os cuidadores, ao assumirem essa tarefa, abrem mão de vários outros aspectos de sua vida, de sonhos e de anseios (FALLER et al., 2012). Para Nortey e colaboradores (2017), o custo mensal total da assistência familiar é elevado, com destaque para o intangível, com alta carga de cuidados e estresse financeiro, sendo que as mulheres expressam uma carga relativamente maior que os homens. 
Para os avanços esperados da AD no âmbito do SUS, é primordial que ensino, pesquisa e prática profissional estejam integrados entre si e ao arcabouço teórico e metodológico das políticas públicas de saúde, pela possibilidade de impacto simultâneo em cada um desses espaços e potencialização do papel dos profissionais de saúde como sujeitos críticos e transformadores (COURA; SILVA; SENA, 2015). Assim, justificam-se estudos e pesquisas que subsidiem novas propostas, revisões e o aprimoramento das políticas que orientam a oferta da AD no contexto da Rede de Atenção à Saúde (RAS).

O desenvolvimento desta pesquisa apoiou-se tanto na prática assistencial de um SAD público vinculado a um hospital de ensino quanto em estudos recentes que reforçam a importância de novas pesquisas voltadas ao fortalecimento da PNAD, à legitimação desta política pública de saúde e à consolidação dos serviços de $\mathrm{AD}$ integrados aos demais serviços da RAS (ANDRADE et al., 2013). Braga e colaboradores (2017) consideram necessária a realização de estudos que contribuam para a análise das potencialidades e das modalidades vigentes de SAD no atendimento das demandas e necessidades de saúde. Com isso, mostra-se relevante captar e compreender as experiências existentes e inscritas ao contexto do SUS, onde a $\mathrm{AD}$ se estabelece como uma nova modalidade assistencial.

Uma questão que inicialmente se levantou relaciona-se ao perfil assistencial dos serviços de $\mathrm{AD}$ que se encontram na condiçâo de serem oferecidos por hospitais públicos, que, embora se orientem pela perspectiva da PNAD, mantêmse com recursos próprios, sem os subsídios do PMC. Outra se refere às condiçôes sociodemográficas e de saúde dos usuários vinculados a esses serviços, pelo potencial de determinarem o perfil assistencial dos mesmos.

$\mathrm{Na}$ revisão prévia da literatura, tomou-se por hipótese que o SAD vinculado a um hospital público de ensino guarda características semelhantes às dos serviços subsidiados pelo PMC, atendendo usuários crônico-dependentes, de baixa renda e com familiares envolvidos no processo do cuidar, para os quais são transferidos custos, gastos e cuidados demandados para a continuidade do atendimento domiciliar. Objetiva-se, portanto, analisar o perfil assistencial de um SAD vinculado a um hospital público de ensino do município do Rio de Janeiro e verificar as associaçôes e correlaçôes entre as variáveis sociodemográficas e de saúde dos usuários assistidos por esse serviço. 


\section{Metodologia}

Este artigo advém da primeira fase da dissertação intitulada Gestão do cuidado domiciliar pelo cuidador de um familiar dependente para o autocuidado, que consistiu em uma pesquisa do tipo documental, com abordagem quantitativa, que teve como cenário um SAD pertencente a um hospital universitário, vinculado ao SUS. O serviço, criado em 1999, é composto por uma equipe multiprofissional com capacidade para atender 50 usuários residentes em uma área programática (AP) do Município do Rio de Janeiro, atendendo tanto adultos quanto idosos nas modalidades AD1 e AD2, e as suas famílias. No entanto, ainda que se oriente pelas diretrizes do SUS e esteja em adequação à PNAD, tal serviço não está cadastrado no PMC por não atender aos requisitos mínimos estabelecidos pela Portaria no 825 de 2016 (BRASIL, 2016).

Os participantes foram todos os usuários com idade igual ou superior a 18 anos cadastrados e atendidos pelo serviço, no período da coleta de dados, totalizando 46 participantes. Portanto, não houve o cálculo da amostra, e sim a participação do universo estatístico. Os dados foram coletados em prontuários por meio de um roteiro estruturado para avaliação do perfil sociodemográfico e de saúde elaborado para esse fim, contendo cinco partes. A primeira visou à obtenção dos dados sociodemográficos; a segunda, à obtenção dos dados relativos à inclusão/elegibilidade e à permanência no $\mathrm{SAD}$, aos equipamentos e aos procedimentos realizados em domicílio; a terceira buscou coletar dados clínicos e a avaliação do grau de dependência para o autocuidado; a quarta, dados sobre as condiçóes de moradia e a composição familiar, e a quinta objetivou levantar dados sociodemográficos dos cuidadores. Este instrumento foi validado por pré-teste, a fim de reduzir a ocorrência dos erros não amostrais e maximizar a confiabilidade e a sua amplitude para a coleta dos dados.

Após o pré-teste, iniciou-se a pesquisa documental, que aconteceu entre os meses de maio e novembro de 2017, com uma média de duas coletas semanais, em sala reservada na própria sede do SAD. Cada coleta em prontuário teve duração média de 60 minutos, devido ao tamanho do prontuário, que continha instrumentos estruturados e evoluçáo da equipe multiprofissional, e do tamanho do roteiro a ser preenchido. Os dados coletados foram organizados em uma planilha idealizada para essa finalidade, usando o programa Excel 2016, do pacote Microsoft. 
A análise dos dados fez-se por meio de estatística básica, composta por medidas de tendência central (média, mediana e moda) e medidas de dispersão (desvio padrão e variância). Além disso, foi aplicado o teste não paramétrico Qui-Quadrado $\left(\chi^{2}\right)$ para análise dos dados categóricos como sexo, grau de dependência para o autocuidado, motivo de inclusão no $\mathrm{SAD}$, classificação na $\mathrm{AD}$, presença de cuidador familiar primário, grau de parentesco do cuidador e renda mensal do usuário. Também foi utilizado o Coeficiente de Correlação de Pearson, com o intuito de medir o grau da correlação linear entre duas variáveis quantitativas. Em todos os testes estatísticos considerados, adotou-se $\alpha$ de 5\% como grau de significância para verificação da rejeição da hipótese de nulidade $(\mathrm{p} \leq 0,05)$. Foi utilizado o programa Excel 2016, do programa Microsoft, e o software Jupyter, para a análise dos dados.

O projeto de pesquisa foi cadastrado na Plataforma Brasil e submetido ao Comitê de Ética em Pesquisa (CEP), atendendo aos princípios éticos, sendo avaliado e autorizado seu início por meio dos pareceres 63887117.2.0000.5147 e 63887117.2.3001.5257.

\section{Resultados e Discussão}

Dos 46 usuários participantes desta pesquisa, 71,74\% eram do sexo feminino e $28,26 \%$ do sexo masculino, com média de idade de 82,76 anos. Com relação ao estado civil, 43,78\% eram viúvos; 34,78\% eram casados ou estavam em união estável, $13,04 \%$ solteiros, e 8,7\% estavam separados ou divorciados. Quanto à escolaridade, os usuários tinham, em média, 5,34 anos de estudo e $60,88 \%$ não possuíam o ensino fundamental completo. Verificou-se, assim, que a maioria dos usuários era idosa com idade avançada e com baixa escolaridade, exigindo da equipe multiprofissional do SAD uma adaptação a este perfil, com adequação das ações a fim de que a comunicação fosse efetiva e a resolubilidade das necessidades de saúde fosse alcançada.

A análise da renda mensal tomou por base o salário-mínimo operado no país em 2018 (R \$ 954). Verificou-se que 65,22\% dos usuários recebiam até dois salários mínimos e apenas $6,52 \%$ possuíam uma renda superior a quatro salários mínimos. Além disso, dos 46 usuários do SAD, 91,3\% recebiam algum benefício assistencial ou previdenciário. Destes, 45,24\% eram aposentados por tempo de serviço, 30,95\% pensionistas, 9,52\% aposentados por invalidez, 7,14\% recebiam o Benefício de Prestação Continuada (BPC) e 7,14\% eram tanto pensionistas quanto aposentados. 
A variável "renda mensal do usuário" teve associação estatisticamente significante com a presença ou ausência de benefício assistencial ou previdenciário $(\mathrm{p}<0,001)$, segundo a tabela 1. Logo, a maior parcela de renda dos usuários do SAD é composta pelos benefícios assistencial ou previdenciário, que compóem um orçamento familiar fragilizado, com altas despesas e baixa receita.

Tabela 1. Associação entre as variáveis "renda mensal do usuário" e a "presença, ou não, do benefício assistencial ou previdenciário”. Rio de Janeiro-RJ, 2018

\begin{tabular}{lcccccc}
\hline \multicolumn{7}{c}{ Renda mensal do usuário* } \\
\hline $\begin{array}{l}\text { Benefício } \\
\text { assistencial ou } \\
\text { previdenciário }\end{array}$ & $\begin{array}{c}\text { De 1 a 2 } \\
\text { SM }^{* *}\end{array}$ & $\begin{array}{c}\text { De 2 a 3 } \\
\text { SM }\end{array}$ & $\begin{array}{c}\text { De 3 a 4 } \\
\text { SM }\end{array}$ & $\begin{array}{c}\text { Maior } \\
\text { que 4 SM }\end{array}$ & Nenhuma & Total \\
Sim & 27 & 10 & 3 & 2 & 0 & 42 \\
Não & 0 & 0 & 0 & 1 & 3 & 4 \\
Total & 27 & 10 & 3 & 3 & 3 & 46 \\
\hline
\end{tabular}

*Teste Qui-Quadrado: $\mathrm{p}<0,001$

**SM=salário-mínimo

Fonte: elaboração própria.

Sobre o motivo de inclusão no SAD, 30,43\% foram admitidos devido à Doença de Alzheimer (DA), 21,74\% por Acidente Vascular Cerebral (AVC), seguido de 8,7\% por fratura de fêmur e/ou outras e $6,52 \%$ por doenças oncológicas. O predomínio dos usuários admitidos por DA tem relação com o aumento do grau de dependência, ocasionado por esta patologia. Tais achados são semelhantes ao encontrado por estudos brasileiros; destacou-se um realizado em Maceió, estado de Alagoas, onde 61,1\% dos pacientes foram admitidos pelo SAD devido a doenças neurológicas, que incluem, também, a associação entre a DA e o AVC (CARNAÚBA et al., 2017).

Além dos diagnósticos admissionais, os pacientes apresentavam comorbidades. É relevante destacar que 61\% possuíam Hipertensão Arterial Sistêmica (HAS); 20\%, DA; 14\%, Diabetes Mellitus (DM); e 13\%, Câncer, patologias crônicas que, quando associadas, podem ampliar as demandas por cuidados de maior complexidade. Estudo realizado por Souza e colaboradores (2014) evidenciou que 54\% dos usuários de um SAD foram diagnosticados por alguma patologia do sistema cardiovascular, em especial a HAS, seguido da DM, o que corrobora os achados desta pesquisa. 
De acordo com o grau de dependência, $10,87 \%$ dos usuários eram independentes, $45,65 \%$ parcialmente dependentes e $43,48 \%$ totalmente dependentes para o autocuidado. A variável "grau de dependência" teve associação estatisticamente significante com o "sexo" $(\mathrm{p}=0,025)$, na qual usuários do sexo feminino apresentavam maior grau de dependência que os do sexo masculino. Tal achado está em concordância com outros estudos (NEVES; CASTRO; COSTA, 2016; MACHADO et al., 2013). Esses dados permitiram inferir que, apesar de haver um maior envelhecimento da população feminina, esta adoece por doenças crônicodegenerativas que reduzem consideravelmente sua independência para realização das atividades de vida diária (AVD) e atividades instrumentais de vida diária (AIVD).

A variável "motivo de inclusão no SAD" teve associação estatisticamente significante com o "grau de dependência" ( $\mathrm{p}=0,003)$, no qual usuários admitidos com DA e AVC eram mais dependentes para o autocuidado, uma vez que tais doenças são mais incapacitantes. Ademais, a variável "especialidade médica que encaminhou ao serviço" também teve associação com o "grau de dependência" ( $p=0,013)$ : usuários encaminhados pela geriatria eram mais dependentes do que os demais, exigindo maior participação da equipe multiprofissional do SAD e de seus cuidadores (tabela 2).

Tabela 2. Associação entre grau de dependência e sexo, presença ou ausência de cuidador familiar primário, motivo de inclusão no $\mathrm{SAD}$, especialidade médica que encaminhou ao SAD e classificação no SAD. Rio de Janeiro-RJ, 2018

\begin{tabular}{llccccc}
\hline Variável & Categorias & $\begin{array}{c}\text { Grau de } \\
\text { dependência } \\
\text { (independente) }\end{array}$ & $\begin{array}{c}\text { Grau de } \\
\text { dependência } \\
\text { (parcialmente } \\
\text { dependente) }\end{array}$ & $\begin{array}{c}\text { Grau de } \\
\text { dependência } \\
\text { (totalmente } \\
\text { dependente) }\end{array}$ & $\chi^{2}$ & P \\
\hline Sexo do & Feminino & 4 & 11 & 18 & 7,34 & 0,025 \\
usuário & Masculino & 1 & 10 & 2 & & \\
\hline $\begin{array}{l}\text { Cuidador } \\
\text { familiar }\end{array}$ & Sim & 3 & 19 & 20 & 8,09 & 0,017 \\
primário & & 2 & 2 & 0 & & \\
\hline
\end{tabular}




\begin{tabular}{|c|c|c|c|c|c|c|}
\hline \multirow{26}{*}{$\begin{array}{l}\text { Motivo de } \\
\text { inclusáo no } \\
\text { SAD }\end{array}$} & AVC & 0 & 5 & 5 & \multirow[t]{26}{*}{52,44} & \multirow[t]{26}{*}{0,003} \\
\hline & Amaurose bilateral & 1 & 0 & 0 & & \\
\hline & \multicolumn{4}{|l|}{ por glaucoma } & & \\
\hline & Artrose e/ou & 1 & 1 & 0 & & \\
\hline & \multicolumn{4}{|l|}{ osteoporose } & & \\
\hline & Câncer & 0 & 1 & 2 & & \\
\hline & DAOP com & 0 & 2 & 0 & & \\
\hline & \multicolumn{4}{|l|}{ amputação } & & \\
\hline & Distrofia muscular & 1 & 0 & 0 & & \\
\hline & progressiva & & & & & \\
\hline & Doença de & 0 & 3 & 11 & & \\
\hline & \multicolumn{4}{|l|}{ Alzheimer } & & \\
\hline & Doença de & 0 & 2 & 0 & & \\
\hline & \multicolumn{4}{|l|}{ Parkinson } & & \\
\hline & Encefalopatia & 0 & 0 & 1 & & \\
\hline & \multicolumn{4}{|l|}{ crônica } & & \\
\hline & Esclerose múltipla & 0 & 1 & 0 & & \\
\hline & \multirow{2}{*}{\multicolumn{2}{|c|}{$\begin{array}{l}\text { Estenose de canal } \\
\text { medular }\end{array}$}} & 1 & 0 & & \\
\hline & & & & & & \\
\hline & $\begin{array}{l}\text { Fratura de fêmur e/ } \\
\text { ou outras }\end{array}$ & 0 & 3 & 1 & & \\
\hline & IAM & 1 & 0 & 0 & & \\
\hline & \multirow{3}{*}{\multicolumn{2}{|c|}{$\begin{array}{l}\text { Síndrome } \\
\text { vertiginosa de } \\
\text { repetição }\end{array}$}} & 1 & 0 & & \\
\hline & & & & & & \\
\hline & & & & & & \\
\hline & Úlcera venosa ou & 1 & 1 & 0 & & \\
\hline & \multicolumn{2}{|l|}{ arterial } & & & & \\
\hline \multirow{8}{*}{$\begin{array}{l}\text { Especialidade } \\
\text { médica que } \\
\text { encaminhou } \\
\text { ao SAD }\end{array}$} & Cardiologia & 2 & 0 & 0 & \multirow[t]{8}{*}{28,39} & \multirow[t]{8}{*}{0,013} \\
\hline & Cirurgia vascular & 0 & 1 & 0 & & \\
\hline & Clínica médica & 1 & 7 & 1 & & \\
\hline & Geriatria & 2 & 11 & 15 & & \\
\hline & Neurologia & 0 & 1 & 1 & & \\
\hline & Oncologia & 0 & 0 & 2 & & \\
\hline & Ortopedia & 0 & 1 & 0 & & \\
\hline & Psiquiatria & 0 & 0 & 1 & & \\
\hline \multirow{3}{*}{$\begin{array}{l}\text { Classificação } \\
\text { no SAD }\end{array}$} & AD1 & 4 & 1 & 0 & \multirow[t]{3}{*}{27,91} & \multirow[t]{3}{*}{$<0,001$} \\
\hline & AD2 & 1 & 20 & 20 & & \\
\hline & AD3 & 0 & 0 & 0 & & \\
\hline
\end{tabular}

Fonte: elaboração própria. 
O predomínio de idosos com dependência para o autocuidado, achados desta pesquisa, colabora para a elevada permanência no SAD, cuja média é de 614,13 dias, causando a estagnação neste ponto da rede, influenciando negativamente a rotatividade de usuários, as filas de espera por vagas e a não abrangência deste serviço para outras áreas geográficas. Para Silva e colaboradores (2017), o perfil da população atendida por um SAD remete à parcela populacional envelhecida, o que requer maior tempo de permanência em atendimento domiciliar, considerando a convivência com doenças crônicas, degenerativas e incapacitantes e, consequentemente, a fragilidade.

Segundo estabelecido pela PNAD e pelo PMC, 10,87\% dos usuários foram classificados em AD1 e 89,13\% em AD2 (BRASIL, 2016). Segundo a Portaria no 825/2016, os usuários classificados em AD1 devem ser atendidos pela APS, porém, conforme citado, pequena parcela dos usuários foi classificada como AD1 (BRASIL, 2016). Nenhum usuário foi classificado em AD3, pois o SAD, cenário desta pesquisa, não admite usuários desta categoria, por não possuir infraestrutura, logística e nem quantitativo de profissionais especializados requeridos para o atendimento desses casos.

Quanto à “classificação na AD", esta variável também demonstrou uma associação estatisticamente significante com o "grau de dependência” ( $\mathrm{p}<0,001)$, onde usuários classificados em AD2 têm maior dependência para o autocuidado. Além disso, a variável "grau de dependência" também teve relação com a "presença ou ausência de cuidador familiar primário" ( $\mathrm{p}=0,017)$ : o maior grau de dependência é acompanhado da presença do cuidador familiar primário (tabela 2). Sendo assim, familiares de usuários com maior dependência para o autocuidado organizam-se de forma a atribuir a função de cuidador a pelo menos um membro da família, alterando ainda mais a dinâmica familiar. Tal organização, por vezes, é exigida de modo repentino, náo permitindo o preparo prévio, o que leva à sobrecarga, demandando dedicação quase que exclusiva ao atendimento das necessidades de saúde do familiar adoecido (NEVES; CASTRO; COSTA, 2016).

Sobre a modalidade de $\mathrm{AD}$, houve predominância de usuários classificados em $\mathrm{AD} 2$, seguidos por $\mathrm{AD} 1$; não se identificando a modalidade $\mathrm{AD} 3$. O percentual de usuários com demandas para a APS (classificados em AD1) é mantido segundo justificativas administrativas relacionadas ao vínculo dos usuários com a equipe, à proximidade geográfica e às condiçôes de funcionamento da APS de referência. 
Estudo realizado em Minas Gerais identificou que, apesar dos critérios de elegibilidade ao SAD estarem definidos pelo Ministério da Saúde, "os gestores e coordenadores abrem exceçôes na inclusão e alta, considerando a fragilidade da rede para alta, encaminhamento para outro ponto de atenção e a realidade clínica ou administrativa do paciente" (PAIVA et al., 2016, p. 251). No serviço pesquisado, apenas 32,61\% dos usuários apresentaram endereço de moradia com cobertura de uma Unidade Básica de Saúde (UBS) com Estratégia de Saúde da Família (ESF). Os demais usuários tinham um Centro Municipal de Saúde (CMS) nas proximidades de sua residência, mas não na modalidade de ESF, o que lhes priva da possibilidade de receberem visitas domiciliares (VD) e receberem acompanhamento concomitantemente por profissionais da APS.

$\mathrm{Na}$ cidade do Rio de Janeiro, no ano de 2009 , apenas $4 \%$ da população estavam cobertos pela ESF, prevalecendo o modelo tradicional da atenção básica na maioria das localidades (JESUS; ENGSTROM; BRANDÁO, 2015). Já em dezembro de 2016, 65\% da população estavam cobertos por uma UBS com ESF (PINTO et al., 2017). Dadas as especificidades de um SAD no contexto da RAS, que tem como centro primário de referência a APS, a ausência da ESF no território dificulta o trabalho conjunto com as equipes multiprofissionais do SAD e, por vezes, os usuários e suas famílias sentem-se "desprotegidos", por não terem uma Unidade de Saúde de referência próxima a seu domicílio, à qual possam recorrer para obter apoio ao cuidado domiciliar (NEVES; CASTRO; COSTA, 2016).

Sobre a associação entre a "classificação no SAD" e o "motivo de inclusão" $(\mathrm{p}<0,001)$, na tabela 3 , compreende-se que as doenças crônicas, degenerativas e paliativas são as mais incapacitantes e que, consequentemente, levam à necessidade de atendimento domiciliar. Tal fato é corroborado por Wachs e colaboradores (2016), que constataram que pessoas com condições crônicas têm um aumento de aproximadamente $40 \%$ de chance de virem a receber atendimento domiciliar. 
Tabela 3. Associação entre as variáveis "classificação no SAD" e "motivo de inclusão no SAD”. Rio de Janeiro-RJ, 2018

\begin{tabular}{lcccc}
\hline Classificação no SAD & & & & \\
\hline Motivo de inclusão no SAD & AD 1 & AD 2 & AD 3 & Total \\
AVC & 0 & 10 & 0 & 10 \\
Amaurose bilateral por glaucoma & 1 & 0 & 0 & 1 \\
Artrose e/ou osteoporose & 2 & 0 & 0 & 2 \\
Câncer & 0 & 3 & 0 & 3 \\
DAOP com amputação & 0 & 2 & 0 & 2 \\
Distrofia muscular progressiva & 0 & 1 & 0 & 1 \\
Doença de Alzheimer & 0 & 14 & 0 & 14 \\
Doença de Parkinson & 0 & 2 & 0 & 2 \\
Encefalopatia crônica & 0 & 1 & 0 & 1 \\
Esclerose múltipla & 0 & 1 & 0 & 1 \\
Estenose de canal medular & 0 & 1 & 0 & 1 \\
Fratura de fêmur e/ou outras & 0 & 4 & 0 & 4 \\
IAM & 1 & 0 & 0 & 2 \\
Síndrome vertiginosa de repetição & 0 & 1 & 0 & 1 \\
Úlcera venosa ou arterial & 5 & 1 & 0 & 2 \\
Total & & 41 & & 4 \\
\hline
\end{tabular}

*Teste Qui-Quadrado: $\mathrm{p}<0,001$

Fonte: elaboração própria.

A média de hospitalizaçóes, no último ano, dos adultos e idosos atendidos pelo SAD pesquisado foi de 0,61, com uma média de 2,22 anos da última internação hospitalar. Esses dados permitem inferir que o serviço atende ao menos um dos objetivos da $\mathrm{AD}$, que é a redução de (re)internaçôes hospitalares, um importante indicador da qualidade assistencial. Estudo de Wachs e colaboradores (2016) analisa que o histórico de hospitalização no último ano apresenta uma forte associação com a $\mathrm{AD}$, na qual usuários em acompanhamento domiciliar apresentam menor número de hospitalizações do que usuários não incluídos no SAD. 
Dos 46 usuários incluídos nesta pesquisa, 8 (17,39\%) evoluíram para óbito e 4 $(8,7 \%)$ receberam alta do serviço. Tal fato demonstra que o óbito prevaleceu como maior motivo de saída do SAD. Acrescenta-se que o óbito no domicílio em usuários atendidos pelo SAD é considerado um fator positivo, demonstrando o bom suporte domiciliar e a não necessidade de hospitalizaçóes. $\mathrm{O}$ cuidador familiar primário estava presente no cuidado de $91,31 \%$ dos usuários do SAD. No PMC a presença do cuidador é pré-requisito para a admissão dos usuários dependentes funcionalmente, de acordo com a Classificação Internacional de Funcionalidade, Incapacidade e Saúde (CIF) (BRASIL, 2016).

Quanto a receber ajuda de outra pessoa, apenas $23,91 \%$ dos usuários contavam com um cuidador secundário e, destes, 45,65\% optaram por contratar um profissional, com ou sem capacitaçáo, para auxiliar o cuidador familiar primário. Quando há a ajuda de outros membros da família ou de um cuidador contratado, a sobrecarga diminui, e este consegue conciliar o cuidado ao outro com o seu autocuidado (NEVES; CASTRO; COSTA, 2016). Porém, o que se pôde constatar é que os cuidadores familiares, em sua maioria, cuidam de forma solitária.

Neste estudo, a maioria dos cuidadores familiares primários era do sexo feminino (85,71\%). Essa predominância segue ao longo do tempo relacionada ao contexto social, cultural, histórico e religioso, além das questôes morais e da "herança familiar", o que ocasiona na "obrigatoriedade" e na aceitação em assumir o papel do cuidado doméstico e familiar (MEIRA et al., 2017).

Os cuidadores familiares primários tinham, em média, 62,3 anos de idade e 9,64 anos de estudo. Ademais, 50\% eram filhas ou filhos e 23,81\% eram cônjuges, mantendo o caráter da consanguinidade, embora já se identifiquem famílias com conformações não consanguíneas. Além disso, a variável "grau de parentesco" teve associação estatisticamente significante com o "sexo" do usuário $(\mathrm{p}=0,045)$ (tabela 4). 
Tabela 4. Associação entre as variáveis "sexo do usuário" e "grau de parentesco do cuidador primário”. Rio de Janeiro-RJ, 2018

\begin{tabular}{lccc}
\hline Grau de parentesco & Feminino & Masculino & Total \\
do cuidador primário* & 3 & 7 & 10 \\
Esposo(a) & 0 & 1 & 1 \\
Ex-esposa & 17 & 4 & 21 \\
Filho(a) & 1 & 0 & 1 \\
Irmáo(ã) & 1 & 0 & 1 \\
Mãe & 3 & 0 & 3 \\
Neto(a) & 3 & 0 & 3 \\
Nora & 1 & 0 & 1 \\
Sobrinho(a) & 1 & 0 & 1 \\
Sogra da filha & 30 & 12 & 42 \\
Total & & & \\
\hline
\end{tabular}

*Teste qui-quadrado: $\mathrm{p}=0,045$

Fonte: elaboração própria.

No ambiente familiar, há determinados critérios relativos à escolha do indivíduo para assumir a função de cuidador, relacionados ao sexo, grau de parentesco, relação com o familiar dependente, personalidade, tempo disponível e se mora na mesma residência ou perto (MAROLDI et al., 2012). Assim, de um modo geral, são as esposas - no caso de usuários do sexo masculino - e as filhas - no caso de usuárias do sexo feminino - que assumem o papel de cuidador familiar primário, por ser sua obrigação manter seu ente sendo cuidado em seu próprio domicílio.

$\mathrm{Na}$ avaliação dos arranjos familiares, identificou-se que, no momento da pesquisa, 57,14\% dos (as) cuidadores (as) estavam casados (as), 19,05\% solteiros (as), 14,29\% viúvos (as) e 9,52\% separados (as) ou divorciados (as). Quanto ao vínculo trabalhista, $69,05 \%$ dos cuidadores familiares eram aposentados (as) e/ou do lar e apenas $30,95 \%$ exerciam alguma atividade remunerada. Esse achado tem relação com o fato de a família priorizar, para assumir esta função, o indivíduo do sexo feminino, casado, que não esteja exercendo nenhuma atividade remunerada, principalmente pela crença de que eles têm mais tempo livre para o cuidado ao outro. Com relação aos 30,95\% que conciliam a função de cuidador com a atividade laborativa, infere-se que se acentua, ainda mais, a sobrecarga física, emocional e social. 
Os usuários do SAD demandavam, em média, 7,46 equipamentos e materiais médico-hospitalares para a continuidade do seu cuidado domiciliar, sendo que $70 \%$ dos gastos relativos a esses equipamentos e materiais eram assumidos pelo usuário e sua família; $10 \%$ de forma compartilhada entre o SAD, o usuário e sua família; 9\% pelo SAD; $5 \%$ por meio de doaçôes e $6 \%$ pelo SUS. Na tabela 5 está apresentada a relação dos equipamentos e materiais médico-hospitalares mais demandados pelos usuários deste SAD.

Tabela 5. Distribuição dos principais equipamentos e materiais médico-hospitalares utilizados por usuários atendidos pelo SAD. Rio de Janeiro-RJ, 2018

\begin{tabular}{lll}
\hline Equipamentos e materiais médico-hospitalares & N & $\%$ \\
\hline Fralda geriátrica & 35 & 76 \\
Luva de procedimentos & 33 & 72 \\
Cobertura para curativos & 26 & 57 \\
Gazes & 25 & 54 \\
Cadeira de rodas & 21 & 46 \\
Soro fisiológico & 21 & 46 \\
Esparadrapo & 21 & 46 \\
Cama hospitalar & 20 & 43 \\
Suplemento nutricional & 20 & 43 \\
Cadeira higiênica & 16 & 35 \\
Esfigmomanômetro & 16 & 35 \\
Termômetro & 8 & 17 \\
\hline
\end{tabular}

Fonte: elaboração própria.

Por meio da Correlação de Pearson, constatou-se que houve diferença estatisticamente significante, correlação positiva, entre os custos e gastos assumidos pelos usuários e suas famílias e o número de equipamentos e materiais médicohospitalares $(\mathrm{p}<0,001)$. Tal achado permitiu inferir que há uma transferência da compra de equipamentos e materiais de saúde para usuários do SAD e sua família, levando a um aumento substancial dos gastos da família com o item saúde, levando à alteração do orçamento familiar e, consequentemente, o aumento da sobrecarga. 
Segundo Silva e colaboradores (2017), os SAD atendem apenas parcialmente às demandas e necessidades de saúde dos usuários e de suas famílias. Para que tais demandas e necessidades de saúde sejam atendidas, torna-se imprescindível o acesso às tecnologias de saúde - leves, leve-duras e duras (MERHY; FEUERWERKER, 2009). Assim, há um repasse de custos e gastos, no qual usuários e famílias assumem, além de custos intangíveis como o isolamento social, os custos diretos, como a compra de insumos, medicamentos e dieta, e os custos indiretos, como a contratação de um cuidador (SILVA et al., 2014; SILVA et al., 2017; FALLER et al., 2012).

De acordo com pesquisa desenvolvida por Souza e colaboradores (2014) quanto às condições de obtenção de materiais de cuidado, 62\% tinham condiçôes financeiras para manter o cuidado domiciliar e 38\% afirmaram que o orçamento familiar era insuficiente para atender ao alto custo do tratamento, além de terem que lidar com a falta de engajamento e de participação dos programas de atenção à saúde da família. Logo, os custos e os gastos elevados com serviços de AD podem ameaçar a economia da família e o bem-estar do usuário, levando à precarização do cuidado, assim como potencializando os riscos de adoecimento do cuidador e da família como um todo (NEVES; CASTRO; COSTA, 2016).

Em conformidade com a transferência de custos e gastos para o usuário e sua família, a pesquisa desenvolvida por Pires e colaboradores (2013) verificou a falta de equipamentos de saúde para o cuidado domiciliar, como materiais para curativos (58,3\%), cama hospitalar $(53,5 \%)$, colchão casca de ovo $(51,2 \%)$, andador $(44,4 \%)$, cadeira de higiene $(32,8 \%)$, cadeira de rodas $(31,7 \%)$ e bengala $(31,25 \%)$.

Segundo os achados desta pesquisa, os usuários do SAD demandavam, em média, 6,78 procedimentos de enfermagem, sendo eles: sinais vitais $(97,8 \%)$; administração de medicamentos (93,5\%); troca de fraldas (71,7\%); transferências (63\%); administração de dieta por via oral (60,9\%); banho de aspersão $(58,7 \%)$; curativo de lesôes (47,8\%); coleta de exames (47,8\%); mudança de decúbito $(37 \%)$; e banho no leito $(28,3 \%)$.

Por meio da Correlação de Pearson, constatou-se que houve diferença estatisticamente significante, correlação positiva, entre os custos e gastos assumidos pelos usuários e suas famílias e o número de procedimentos de enfermagem demandados pelos usuários do $\mathrm{SAD}(\mathrm{p}<0,001)$ e entre o número de dispositivos invasivos e o número de procedimentos de enfermagem demandados pelos usuários do SAD ( $\mathrm{p}<0,001)$. Diante disso, inferiu-se que, apesar de o contexto domiciliar 
ser um local singular, repleto de significados e sentimentos, há um predomínio no uso das tecnologias duras, representadas pelo uso de equipamentos de saúde, em detrimento das tecnologias leves - que valorizam o relacionamento interpessoal e o estabelecimento de vínculos, e das tecnologias leve-duras, baseadas nos conhecimentos já estruturados (MERHY; FEUERWERKER, 2009).

Do total de procedimentos de enfermagem, 52\% eram realizados pelo próprio cuidador, $23 \%$ pelos profissionais da equipe multiprofissional, $13 \%$ realizados de forma compartilhada entre o SAD e o cuidador, $10 \%$ realizados de forma conjunta entre o cuidador e o próprio usuário, e $1 \%$ pelo serviço privado e o SAD. A partir disso, compreende-se a importância do atendimento integral de usuários e familiares pelo SAD, oferecendo cuidados de enfermagem de acordo com as suas necessidades de saúde e/ou realizando educação em saúde de modo a capacitar o cuidador para a sua realização (NEVES; CASTRO; COSTA, 2016).

Os cuidadores familiares assumem uma multiplicidade de tarefas, sendo que algumas delas representam procedimentos que são privativos da enfermagem, como, por exemplo, o cateterismo vesical, o tratamento de lesóes e a coleta de exames. Tal situação leva ao aumento considerável da sobrecarga do cuidador e afeta negativamente o cuidado prestado a seu familiar, já que o mesmo não possui habilidades e competências condizentes com essas funçóes e responsabilidades (SILVA et al., 2014).

Para Neves, Castro e Costa (2016), é papel do Estado a ampliação do acesso à $\mathrm{AD}$, com a prestação de cuidados de qualidade, de forma que náo seja transferida aos cuidadores toda a responsabilidade pelo usuário, tanto na execução dos cuidados quanto na transferência de custos e gastos. O Estado deve assumir o compartilhamento que lhe cabe, assegurando os itens cujo acesso é garantido pela Constituição Federal e por políticas públicas específicas.

Ainda sobre as necessidades de saúde atendidas no âmbito dos SAD, uma pesquisa desenvolvida na Espanha por Gascón e López (2017) evidenciou que estas estão mais frequentemente relacionadas à higiene pessoal (72\%), ao apoio à mobilidade (46\%), à manutenção da casa e ao acompanhamento integral (44\%). Tais resultados estáo em concordância com os achados desta pesquisa. Depreende-se que famílias se estruturam para manter o cuidado de seu ente em domicílio e, nos casos em que tais usuários demandam um maior número de procedimentos de enfermagem, há o uso mais frequente de equipamentos e materiais médico-hospitalares e, consequentemente, maiores custos e gastos a serem assumidos. 


\section{Considerações finais}

Esta pesquisa demonstrou que os usuários em atendimento por um serviço de $\mathrm{AD}$ vinculado a um hospital público de ensino possuem diferentes e elevadas necessidades e demandas de saúde a serem atendidas pela equipe multiprofissional do SAD em conjunto com cuidadores. Entretanto, constatou-se uma transferência de funçôes e responsabilidades para a família, além de custos e gastos, necessários para o provimento e a manutenção do cuidado domiciliar.

Os cuidadores são, principalmente, familiares, igualmente idosos, sendo filhos(as) ou esposos(as), com baixa escolaridade, em condiçóes de aposentados ou do lar e dedicados, quase que exclusivamente, ao cuidado do familiar em domicílio. Os usuários atendidos convivem com doenças crônicas e degenerativas, que os caracterizam como parcialmente ou totalmente dependentes para o autocuidado, sendo, em sua maioria, idosos com idade avançada, do sexo feminino, com baixa escolaridade, orçamento familiar fragilizado, classificados em AD2 e recebendo a assistência do SAD por um longo período.

Considera-se de extrema relevância a revisão da PNAD à luz dos achados desta e de outras pesquisas, assim como a realização de estudos que procurem dar conta de outros aspectos e cenários, que apontam para a sobrecarga gerada pela transferência de custos e de cuidados técnicos para o cuidador familiar de usuários em atendimento domiciliar, sobretudo no âmbito do SUS.

Assim, torna-se necessário o investimento governamental na ampliação, aprimoramento e fortalecimento da AD como modelo substitutivo e complementar de cuidado, pelos benefícios já constatados. Dessa forma, seria possível às equipes prestar um atendimento integral ao usuário, mas também aos demais participantes do processo de cuidar, em especial aos cuidadores familiares, diminuindo a sobrecarga física, emocional, financeira e social dessas famílias. ${ }^{1}$

\section{Referências}

ANDRADE, A. M. et al. Singularidades do trabalho na Atençâo Domiciliar: imprimindo uma nova lógica em saúde. Rev. Pesq.: Cuidado é Fundamental Online. Rio de Janeiro, v. 5, n. 1, p. 3.383-3.393, jan./mar. 2013.

ANDRADE, A. M. et al. Atuação do enfermeiro na atenção domiciliar: uma revisão integrativa da literatura. Rev. Bras. Enferm. Brasília, v. 70, n. 1, p. 210-9, jan./fev. 2017. 
ANJOS, A. C. Y.; ZAGO, M. M. F. Ressignificação da vida do cuidador do paciente idoso com câncer. Rev. Bras. Enferm. v. 67, n. 5, p. 752-8, out. 2014.

BRAGA, P. P. et al. Oferta e demanda na atenção domiciliar em saúde. Ciência \& Saúde Coletiva. Rio de Janeiro, v. 21, n. 3, p. 903-912, 2017.

BRASIL. Portaria no 2029, de 24 de agosto de 2011. Institui a Atenção Domiciliar no âmbito do Sistema Único de Saúde (SUS). Diário Oficial da União, Brasília, DF, 25 ago. 2011. Seção 1, p. 91. Disponível em:< http://bvsms.saude.gov.br/bvs/saudelegis/gm/2011/prt2029_24_08_2011. html>. Acesso em: 20 nov. 2017.

. Portaria no 825, de 25 de abril de 2016. Redefine a Atenção Domiciliar no âmbito do Sistema Único de Saúde (SUS) e atualiza as equipes habilitadas. Diário Oficial da União, Brasília, DF, 26 abr. 2016. Seção 1, p. 33-38. Disponível em: <http://u.saude.gov.br/images/ pdf/2016/abril/27/PORTARIA-825.pdf>. Acesso em: 14 nov. 2017.

. Ministério da Saúde. Avaliação econômica em saúde: desafios para gestão no Sistema Único de Saúde. Brasília, 2008. Disponível em: <http://bvsms.saude.gov.br/bvs/publicacoes/ avaliacao_economica_desafios_gestao_sus.pdf>. Acesso em: 10 abr. 2017.

CARNAÚBA, C. M. D et al. Caracterização clínica e epidemiológica dos pacientes em atendimento domiciliar na cidade de Maceió, AL, Brasil. Rev. Bras. de Geriatria e Gerontologia. Rio de Janeiro, v. 20, n. 3, p. 353-63, mai./jun. 2017.

CASTRO, E. A. B. et al. Organização da atenção domiciliar com o Programa Melhor em Casa. Rev. Gaúcha Enferm. Porto Alegre, v. 39, p. 1-20, 2018.

COURA, K. R. A.; SILVA, K. L.; SENA, R. R. A formação do enfermeiro em relação às políticas de saúde na expansão do ensino superior. Rev. Enferm. UFPE, Recife, v. 9, n. 5, p. 7826-7834, maio 2015.

FALLER, J. W. et al. Sobrecarga e mudanças no cotidiano de cuidadores familiares de paciente com doença crônica. Ciênc. Cuid. Saúde, v. 11, n. 1, p. 181-9. jan.-mar. 2012.

GASCÓN, M. L. G.; LÓPEZ, L. G. Personas usuarias del Servicio de Ayuda a Domicilio. Una mirada desde la vulnerabilidad y la fragilidad. Index de Enfermería. Granada, v. 26, n. 3, p. 210-214, 2017.

JESUS, R. L.; ENGSTROM, E.; BRANDÃO, A. L. A expansão da Estratégia Saúde da Família no Rio de Janeiro, 2009-2012: estudo de caso numa área da cidade. Rev. Bras. Medicina de Família e Comunidade, v. 10, n. 37, p. 1-11, out.-dez. 2015.

LIMA-COSTA, M. F. et al. Cuidado informal e remunerado aos idosos no Brasil (Pesquisa Nacional de Saúde, 2013). Rev. Saúde Pública, v. 51, n. supl. 1, p. 1-9, 2017.

LOUREIRO, L. S. N.; FERNANDES, M. G. M. Perfil do cuidador familiar de idosos dependentes em convívio domiciliar. Rev. Pesq. Cuidado é Fundamental, v. 7, n. supl., p. 145-154, dez. 2015. 
MACHADO, A. L. G. et al. Estudo dos fatores sociodemográficos associados à dependência funcional em idosos. Rev. Enferm. UFPI, v. 2, n. 1, p. 44-8, 2013.

MAROLDI, M. A. C. et al. Internação domiciliar: caracterização de usuários e cuidadores. CuidArte, Enferm., v. 6, n. 1, p. 24-29, jan.-jun. 2012.

MEIRA, E. C. et al. Vivências de mulheres cuidadoras de pessoas idosas dependentes: orientação de gênero para o cuidado. Esc. Anna Nery. Rio de Janeiro, v. 21, n. 2, p., 1-8, maio 2017.

MERHY, E. E.; FEUERWERKER, L. C. M. Novo olhar sobre as tecnologias de saúde: uma necessidade contemporânea. In: MANDARINO, A. C. S.; GOMBERG, E. (Orgs.). Leituras de novas tecnologias e saúde. São Cristóvão: Editora UFS, 2009. 285 p.

MESQUITA, S. R. A. M. et al. Programa Interdisciplinar de internaçáo domiciliar de Marília- SP: custos de recursos materiais consumidos. Rev. Latino-Am. Enfermagem, v. 13, n. 4, p. 555-61, 2005.

MOCELIN, C. et al. O cuidado do idoso dependente no contexto familiar. Rev. Fun. Care Online, v. 9, n. 4, p. 1034-9, out./dez. 2017.

NEVES, A. C. O. J.; CASTRO, E. A. B.; COSTA, S. R. D. Necessidades de cuidados domiciliares de enfermagem após a alta hospitalar no contexto do SUS. Cogitare Enferm., v. 21, n. 4, p. 01-10, out.-dez. 2016.

NORTEY, S.T. et al. Economic burden of family caregiving for elderly population in southern Ghana: the case of a peri-urban district. International Journal for Equity in Health, v. 16, n. 16, 2017.

OLIVEIRA, M. L.; SANTOS, L. M. P.; SILVA, E. N. Bases metodológicas para estudos de custos da doença no Brasil. Rev. Nutr. Campinas, v. 27, n. 5, p. 585-95, set./out. 2014.

PAIVA, P. A. et al. Serviços de atenção domiciliar: critérios de elegibilidade, inclusão, exclusão e alta. Rev. Bras. Promoção da Saúde, v. 29, n. 6, p. 244-252, 2016.

PINTO, L. F. et al. Sistemas Comparados de Saúde: Atenção Primária à Saúde nas cidades de Lisboa e do Rio de Janeiro. Ciência \& Saúde Coletiva. Rio de Janeiro, v. 22, n. 3, p. 676-677, 2017.

PIRES, M. R. G. M. et al. Fatores associados à atenção domiciliária: subsídios à gestão do cuidado no âmbito do SUS. Rev. da Escola de Enferm. da USP. São Paulo, v. 47, n. 3, p. 648656, jun. 2013.

SILVA, K. L. et al. Desafios da atenção domiciliar sob a perspectiva da redução de custos/ racionalização de gastos. Rev. Enferm. UFPE. Recife, v. 8, n. 6, p. 1561-7, jun. 2014.

SILVA, K. L. et al. Por que é melhor em casa? A percepção de usuários e cuidadores da Atenção Domiciliar. Cogitare Enferm., v. 22, n. 4, p. 1-9, 2017. 
SOLÉ-AURÓ, A.; CRIMMINS, E. M. Who cares? A comparison of informal and formal care provision in Spain, England and the USA. Ageing Society, v. 34, n. 3, p. 495-517, mar. 2014.

SOUZA, I. C. et al. Perfil de pacientes dependentes hospitalizados e cuidadores familiares: conhecimento e preparo para as práticas do cuidado domiciliar. Rev. Mineira Enferm. Belo Horizonte, v. 18, n. 1, p. 173-80, jan./mar. 2014.

VAZ, L. C. S.; SANTOS, K. O. B.; FERRAZ, D. D. Condições de saúde e trabalho entre cuidadores de idosos frágeis. Rev. Pesquisa em Fisioterapia, v. 8, n. 3, p. 319-329, 2018.

WACHS, L. S. et al. Prevalência da assistência domiciliar prestada à população idosa brasileira e fatores associados. Cad. Saúde Pública. Rio de Janeiro v. 32, n. 3, p. 1-9, mar. 2016.

\section{Nota}

${ }^{1}$ A.C.O.J. Neves realizou a concepção e construção do projeto de pesquisa; coleta de dados; análise e interpretação dos dados; redação do artigo; e aprovação final da versão a ser publicada. C. T. Seixas e A. M. Andrade realizaram a análise e interpretação dos dados; revisão crítica relevante do conteúdo intelectual e aprovaram a versão final a ser publicada. E. A. B. Castro foi responsável pela concepçáo e construção do projeto de pesquisa; análise e interpretação dos dados; revisão crítica relevante do conteúdo intelectual; e aprovação final da versão a ser publicada. As autoras informam que esta pesquisa não recebeu financiamento e que não existem conflitos de interesse. 


\section{Abstract}

Home care: care profile of a service linked to a teaching hospital

Objectives: To describe the profile of the users and caregivers attended by the Home Care Service; to verify associations and correlations among variables. Method: Documentary, quantitative study conducted between May and November 2017. Data analysis was performed using basic statistics, chi-square test and Pearson's correlation coefficient. Results: Of the 46 users, most were elderly, female (71.74\%), with income of up to two minimum wages $(65.22 \%)$, dependent for self-care (89.13\%). The variable "degree of dependence" had a statistically significant association with sex $(p=0.025)$, inclusion motive $(p=0.003)$, medical specialty $(\mathrm{p}=0.013)$, SAD classification $(\mathrm{p}$ $<0.001)$ and presence of caregiver $(\mathrm{p}=0.017)$. They demanded 7.46 health equipment, $70 \%$ of which were housed by the family; and 6.78 nursing procedures, of which $52 \%$ were performed by the caregiver. There was a statistically significant difference between the costs and expenses assumed by the families ( $p<0.001)$, the number of health facilities $(\mathrm{p}<0.001)$ and the number of nursing procedures required ( $\mathrm{p}<0.001)$. Among caregivers, most of them were female (85.71\%), with a mean age of 62.3 years, $50 \%$ were children and $23.81 \%$ were spouses. Conclusion: Functions, responsibilities, costs and expenses are transferred to families, burdening home care.

Keywords: home care; caregivers; health services needs and demand; healthcare costs. 